\title{
Slip Flow of Kerosene Oil Based SWCNT Nanofluid over Stretching Sheet with Radiation and Suction/Injection Effects
}

\author{
Susheela Chaudhary \\ Department of Mathematics, \\ Government Science College, Sikar-332001, India. \\ E-mail: susheelamaths@gmail.com \\ Kiran Kunwar Chouhan \\ Department of Mathematics, \\ Malaviya National Institute of Technology, Jaipur-302017, India. \\ E-mail: kiranchouhanudr@gmail.com \\ Santosh Chaudhary \\ Department of Mathematics, \\ Malaviya National Institute of Technology, Jaipur-302017, India. \\ Corresponding author: d11.santosh@yahoo.com
}

(Received on February 4, 2021; Accepted on April 28, 2021)

\begin{abstract}
Present study numerically investigates a two dimensional steady laminar boundary layer nanofluid flow of single-wall carbon nanotubes (SWCNTs) immersed into kerosene oil, due to a linearly stretched sheet. Flow is subjected to the slip boundary condition and suction/injection effects. Employing suitable similarity transformations, governing PDEs of the arising problem are converted into coupled nonlinear non-dimensional ordinary differential equations. A set of obtained ODEs with assisting boundary conditions is solved numerically by applying finite element method (FEM). Effect of pertinent factors, velocity slip parameter, suction/injection parameter and solid volume fraction parameter on nondimensional velocity and temperature profiles are characterized graphically. In addition, physical emerging parameters, local Nusselt's number and local skin friction coefficient are computed and presented via table. Furthermore, derived numerical values of shear stress and heat flux at the surface are compared with previously published results.
\end{abstract}

Keywords- Slip condition, Nanofluids, Stretching surface, Thermal radiation, Suction/injection.

\section{Introduction}

Slip effect takes place when cohesive forces between fluid particles dominate over adhesive forces between fluid particles and solid surface and normal velocity becomes non-zero. Usually, most of the analyses are performed with no-slip boundary conditions by considering Knudsen number as zero $(K n=0)$, but for some physical situations involving emulsions, suspensions, foams and polymer solutions, the no-slip boundary condition is no longer justifiable. Therefore, velocity slip and temperature jump can be materialized for the ranges of Knudsen number, $10^{-3}<K n<0.1$ (slip flow). Slip flow is of considerable significance for its numerous applications in field of technology and industry like polishing valves of artificial heart and internal cavities. Jang and Wereley (2004) proposed an analytical expression for pressure distribution of gas in a uniform and straight rectangular micro-channel in the slip flow regime. A numerical investigation was carried out by Mukhopadhyay and Andersson (2009) on heat transfer and unsteady viscous flow towards a stretching surface with impact of slip condition. In recent 
International Journal of Mathematical, Engineering and Management Sciences

Vol. 6, No. 3, 860-877, 2021

https://doi.org/10.33889/IJMEMS.2021.6.3.051

years, Hayat et al. (2011), Akbar et al. (2014), Shaw et al. (2016), Afify (2017) and Ghosh and Mukhopadhyay (2019) explored the slip effect on viscous flow of various nanofluids.

Nanotechnology is the branch of science, which is devoted to investigation of physical and thermal attributes of atoms and molecules at nanoscale. Nanofluid is one of the most widely used technique of nanoscience in field of heat transfer phenomenon. The term "nanofluid" interprets dilute mechanical suspension of metallic or non-metallic nanometre sized particle in customary fluids. The idea of nanofluids was propounded by Choi and Eastman (1995), later on, it fascinated many scientists and researchers for its efficient thermal properties. Nanofluids have secured noteworthy applications, namely, aerospace tribology, cooling of microchips, medical suspension, sterilization, powder energy, nuclear reactors, geothermal energy extraction and many such automotive, electronics and biomedical applications. Nanoparticles usually constitute graphite and carbon nanotubes (CNTs), where carbon nanotubes are typical cylindrical shaped molecules comprising rolled-up sheets of carbon atoms. Generally, two types of carbon nanotubes, single walled carbon nanotubes (SWCNTs) and multi-walled carbon nanotubes (MWCNTs) are used. CNTs play a vital role in the field of nanotechnology due to its higher electrical and thermal conductivity and mechanical strength. Xie et al. (2003) investigated the enhancement of thermal conductivity through MWCNTs nanofluid and detected that thermal conductivity of CNTs depends on interfacial layers between the fluids and nanotubes. An overview on heat transfer and convective flow of carbon nanotubes based aqueous drag-reducing fluid was given by Liu and Liao (2010). Kumaresan and Velraj (2012) experimentally analysed the thermo-physical characteristics of CNTs based nanofluid with host fluid water-ethylene glycol. Haq et al. (2015) examined convective heat transfer and MHD flow of both SWCNTs and MWCNTs based nanofluid past by a stretching surface with slip effect. Chaudhary and Kanika (2019) numerically explored a Marangoni convective MHD flow of CNTs nanofluid with radiation effects. Recently, Tulu and Ibrahim (2020) discussed a magneto-hydrodynamic slip flow of CNT-EG nanofluid over a rotating stretchable disk along Cattaneo-Christov heat flux model.

In past few decades, investigations on boundary layer flow due to a stretching surface have acquired abundant interest of researchers for its promising applications in engineering, metallurgical, industrial and manufacturing processes. For instance, crystal growing, aerodynamic extrusions of plastic and plastic sheets, wire drawing, glass blowing, hot rolling and paper production. Crane (1970) pioneered the analysis of a two-dimensional boundary layer flow over a stretching surface and later on, this work is extended by many researchers with MHD, heat and mass transfer, sole effect of rotation, non-Newtonian fluid and many more feasible combinations of various effects. Cortell (2005) established boundary layer flow and heat transfer in porous medium caused by a stretching surface. Mukhopadhyay and Layek (2008) considered a free convective flow of a fluid over a stretching surface under the impact of thermal radiation and irregular fluid viscosity. Akbar et al. (2013) studied a steady two-dimensional magnetohydrodynamic stagnation point flow of nanofluid past a stretching surface along thermal radiation effect and convective boundary condition. Gireesha et al. (2016) carried out a theoretical investigation on heat transfer in dusty fluid flow in porous medium over a nonisothermal stretching surface with radiation and Hall effects. Recently, Mishra and Kumar (2020) discussed thermal and velocity slip on MHD flow of a nanofluid towards a stretching cylinder.

Thermal radiation is a mode of heat transfer mechanism through which emission of thermal energy takes place in form of electromagnetic waves at the surface of matter with nonzero absolute temperature. In other words, it is a transformation of thermal energy into 
electromagnetic energy which is completely independent of any material medium. An analysis on radiation effects on magneto-hydrodynamic mixed free convective flow over a moving semiinfinite plate was carried out by Azzam (2002). Rad and Aghanajafi (2009) studied the thermal radiation impact on nanofluid flow in a rectangular micro-channel. Subsequently, several researchers, such as Hady et al. (2012), Sheikholeslami and Ganji (2015), Dogonchi and Ganji (2017), Eid and Makinde (2018), Krishna et al. (2019) and recently, Kanika et al. (2020) introduced problems concerning radiation effect on nanofluid flow.

Boundary layer suction in amongst boundary layer controlling techniques proposed by L. Prandtl in 1904, in an effort to slow down the boundary layer separation by reducing drag on the bodies in exterior flow. Suction and injection are of common concern in practical problems relating to aerodynamics and space sciences. El-Arabawy (2003) analysed suction and injection effects on micro-polar fluid flow over a continuously moving plate in presence of thermal radiation. Attia (2007) used uniform suction and injection impact on MHD flow of a fluid through rotating disk with heat transfer, Joule heating and Hall effects. Kandasamy et al. (2011) considered suction/injection effect on MHD nanofluid flow due to a stretching surface and observed that higher suction effect leads to decrease velocity and temperature profiles. Makinde and Chinyoka (2013), Khan et al. (2016), Mohammadein et al. (2018) and Chaudhary and Kanika (2020) also used suction and injection influence on various boundary layer flows.

In view of the above literatures, boundary layer flows of nanofluids, induced by stretching surface have been frequently addressed in recent studies. However, radiation and suction/injection impact on SWCNT-kerosene oil nanofluid over stretching sheet with slip boundary condition has still not been investigated. In this study, the numerical solution has been attained for the momentum and energy equations by implementing finite element method and results are portrayed graphically to interpret the behaviour of pertinent parameters. Moreover, the results of this study can lead to further improvements in the efficiency of heat exchangers and solar collectors systems (Ghalandari et al., 2020).

\section{Mathematical Formation}

\subsection{Problem Statement}

To develop the model, consider a steady two-dimensional viscous flow of an incompressible nanofluid induced by a stretching surface under slip boundary condition and uniform suction/injection effects, as shown in Figure 1. The nanofluid consists of SWCNTs with host fluid kerosene oil. It is assumed that host fluid and nano-tubes are in thermal equilibrium. Moreover, the thermal radiation effect is taken into consideration and viscous dissipation is considered as negligible in this study. The flow occupies the semi-infinite plane $y>0$, where $x$-axis is aligned with the direction of the flow and stretching sheet, whereas $y$-axis is measured normal to it. The stretching rate of the sheet is taken as the linear function of the $x$, that is $u_{s}(x)=b x . T$ and $T_{\infty}$ signify the temperature of the nanofluid around boundary layer and far from the wall, $T_{s}(x)=T_{\infty}+c x$ is the surface temperature of the stretching sheet, where $c$ is a constant parameter. Due to aforementioned considerations, the system of elementary equations are specified as follows:

$$
\frac{\partial u}{\partial x}+\frac{\partial v}{\partial y}=0
$$


$u \frac{\partial u}{\partial x}+v \frac{\partial u}{\partial y}=v_{n f} \frac{\partial^{2} u}{\partial y^{2}}$

$u \frac{\partial T}{\partial x}+v \frac{\partial T}{\partial y}=\alpha_{n f} \frac{\partial^{2} T}{\partial y^{2}}-\frac{1}{\left(\rho C_{p}\right)_{n f}} \frac{\partial q_{r}}{\partial y}$

subjected to the following boundary conditions:

$$
\begin{aligned}
& y=0: u=u_{s}+u_{s l i p}, v=v_{s}, T=T_{s}(x) \\
& y \rightarrow \infty: u \rightarrow 0, T \rightarrow T_{\infty}
\end{aligned}
$$

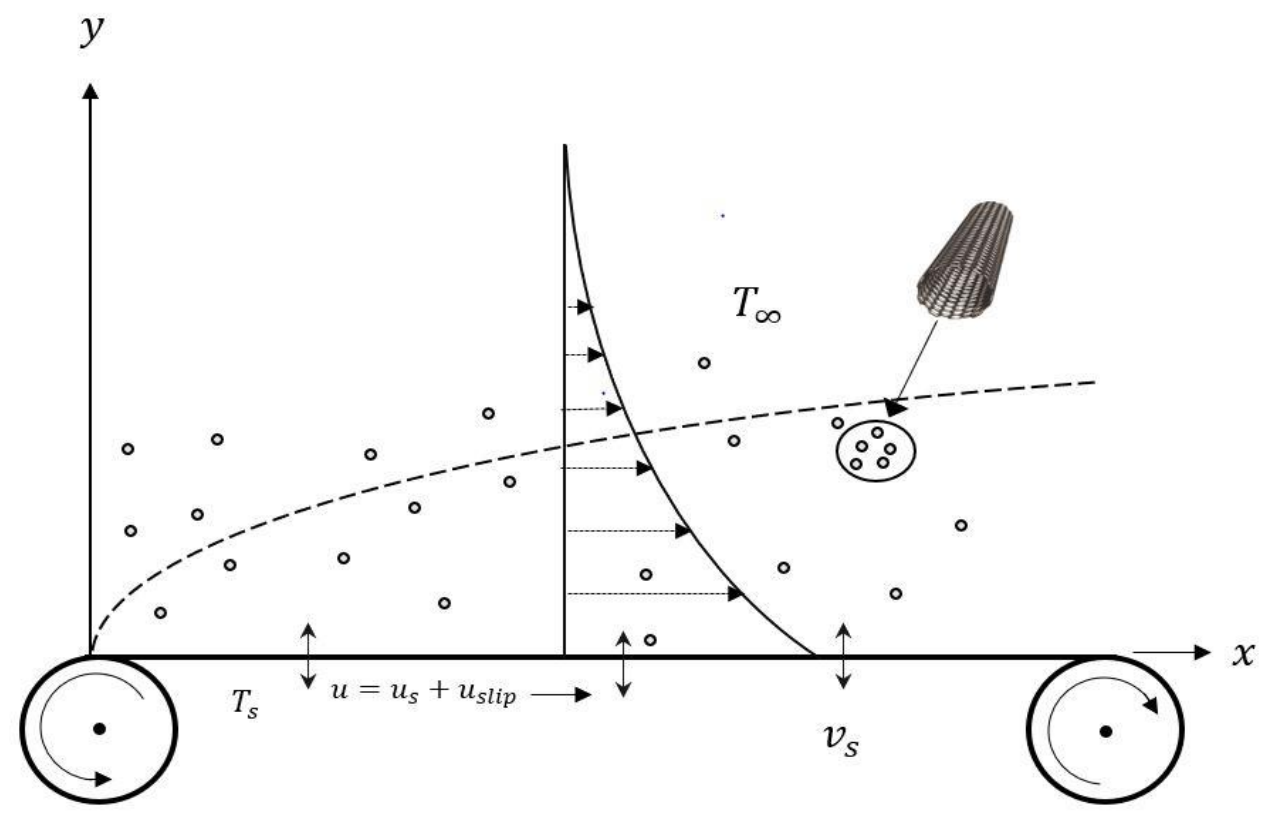

Figure 1. Schematic view of the flow.

where $u$ and $v$ are the velocity constituents in $x$ and $y$ - axes directions, subscript $n f$ refers nanofluid, $v\left(=\frac{\mu}{\rho}\right)$ is the kinematic viscosity, $\mu$ is the dynamic viscosity, $\rho$ is the density, $\alpha\left(=\frac{\kappa}{\rho C_{p}}\right)$ is the thermal diffusivity, $\kappa$ is the thermal conductivity, $C_{p}$ is the specific heat at constant pressure, $q_{r}$ is the radiative heat flux, $u_{\text {slip }}\left(=l \frac{\partial u}{\partial y}\right)$ is the slip velocity, $l$ is the slip 
length and $v_{s}$ is the velocity of mass transfer due to uniform suction $\left(v_{s}>0\right)$ or injection $\left(v_{s}<0\right)$ normal to the stretching sheet.

The Rosseland approximation (Pantokratoras and Fang, 2012) is utilized to simplify the radiative heat flux as $q_{r}=-\frac{4 \sigma^{*}}{3 k^{*}} \frac{\partial T^{4}}{\partial y}$. Subsequently, $\frac{\partial q_{r}}{\partial y}$ is obtained as $-\frac{16 \sigma^{*} T_{\infty}^{3}}{3 k^{*}} \frac{\partial^{2} T}{\partial y^{2}}$ by use of

Taylor series expansion.

\subsection{Nanofluid Properties}

The physical parameters for SWCNT-kerosene oil nanofluid, namely, dynamic viscosity $(\mu)$, density $(\rho)$, thermal conductivity $(\kappa)$ and heat capacity $\left(\rho C_{p}\right)$ are expressed as follows:

$$
\begin{aligned}
& \mu_{n f}=\frac{1}{(1-\phi)^{5 / 2}} \mu_{f} \\
& \rho_{n f}=\left(1-\phi+\phi \frac{\rho_{C N T}}{\rho_{f}}\right) \rho_{f}
\end{aligned}
$$

$\kappa_{n f}=\frac{1-\phi-2 \phi \frac{\kappa_{C N T}}{\kappa_{f}-\kappa_{C N T}} \ln \frac{\kappa_{f}+\kappa_{C N T}}{2 \kappa_{f}}}{1-\phi-2 \phi \frac{\kappa_{f}}{\kappa_{f}-\kappa_{C N T}} \ln \frac{\kappa_{f}+\kappa_{C N T}}{2 \kappa_{f}}} \kappa_{f}$

$$
\left(\rho C_{p}\right)_{n f}=\left[1-\phi+\phi \frac{\left(\rho C_{p}\right)_{C N T}}{\left(\rho C_{p}\right)_{f}}\right]\left(\rho C_{p}\right)_{f}
$$

here $f$ and $C N T$ are subscripts representing base fluid and nano-sized particles and $f$ is the volume fraction of nanotubes. Moreover, the thermo-physical properties of nanotubes and base fluid are presented in the Table 1 (Hayat et al., 2016).

Table 1. Thermo-physical properties of nanotubes and base fluid.

\begin{tabular}{|c|c|c|c|}
\hline Properties & $\rho\left(\mathrm{Kg} \mathrm{m}^{-3}\right)$ & $\kappa\left(\mathrm{Wm}^{-1} \mathrm{~K}^{-1}\right)$ & $C_{p}\left(\mathrm{JKg}^{-1} \mathrm{~K}^{-1}\right)$ \\
\hline SWCNT & 2600 & 6600 & 425 \\
\hline Kerosene Oil & 783 & 0.145 & 2090 \\
\hline
\end{tabular}

\subsection{Similarity Conversion}

The following dimensionless variable are to be utilized to transform the above mathematical model in non-dimensional form (Jafari and Freidoonimehr, 2015). 
$\psi=\left(v_{f} b\right)^{1 / 2} x f(n), \quad \eta=\left(\frac{b}{v_{f}}\right)^{1 / 2} y, \quad T=\left(T_{s}-T_{\infty}\right) \theta(\eta)+T_{\infty}$

where, stream function $\psi(x, y)$ is such that $u=\frac{\partial \psi}{\partial y}$ and $v=-\frac{\partial \psi}{\partial x}$, that identically satisfies the continuity equation (1). Therefore, the resulting non-linear dimensionless ordinary differential equations, by plugging the above variables in equations (2)-(4), are obtained as follows:

$$
\begin{aligned}
& \frac{1}{(1-\phi)^{5 / 2}} f^{\prime \prime \prime}+\left(1-\phi+\phi \frac{\rho_{C N T}}{\rho_{f}}\right)\left(f f^{\prime \prime}-f^{\prime 2}\right)=0 \\
& \left(N r+\frac{\kappa_{n f}}{\kappa_{f}}\right) \theta^{\prime \prime}+\left[1-\phi+\phi \frac{\left(\rho C_{p}\right)_{C N T}}{\left(\rho C_{p}\right)_{f}}\right] \operatorname{Pr}\left(f \theta^{\prime}-f^{\prime} \theta\right)=0
\end{aligned}
$$

associated with boundary conditions

$$
\begin{aligned}
& \eta=0: f=S, f^{\prime}=1+\delta f^{\prime \prime}, \theta=1 \\
& \eta \rightarrow \infty: f^{\prime} \rightarrow 0, \theta \rightarrow 0
\end{aligned}
$$

In the above equations, primes represent derivatives with respect to similarity variable $\eta, f$ is the dimensionless stream function, $\theta$ is the dimensionless temperature, $N r\left(=\frac{16 \sigma^{*} T_{\infty}^{3}}{3 k^{*} \kappa_{f}}\right)$ is the radiation parameter, $\operatorname{Pr}\left[=\frac{v_{f}\left(\rho C_{p}\right)_{f}}{\kappa_{f}}\right]$ is the Prandtl number, $S\left[=-\frac{v_{s}}{\left(v_{f} b\right)^{1 / 2}}\right]$ is the mass flux parameter and $\delta\left[=l\left(\frac{b}{v_{f}}\right)^{1 / 2}\right]$ is the velocity slip parameter. It is noteworthy that $S$ is positive for suction and negative for injection process.

\section{Parameters of Engineering Interest}

\subsection{Skin Friction Coefficient}

The statement of local skin friction coefficient $C_{f}$ is given by:

$$
C_{f}=\frac{\tau_{s}}{\frac{\rho_{f} u_{s}^{2}}{2}}
$$


where $\tau_{s}=\mu_{n f} \frac{\partial u}{\partial y}$ is the shear stress at the sheet, i.e., at $y=0$. Implementing the nondimensional variables of equation (9) into equation (13), the parameter is evaluated as:

$C_{f} \operatorname{Re}_{x}^{1 / 2}=\frac{2}{(1-\phi)^{5 / 2}} f^{\prime \prime}(0)$

where $\operatorname{Re}_{x}=\frac{u_{s} x}{v_{f}}$ is the local Reynolds number.

\subsection{Nusselt Number}

The local Nusselt number $N u_{x}$ is expressed as follows:

$N u_{x}=\frac{x q_{s}}{\kappa_{f}\left(T_{s}-T_{\infty}\right)}$

here $q_{s}\left(=-\kappa_{n f} \frac{\partial T}{\partial y}+q_{r}\right)$ is the heat flux at surface, i.e., at $y=0$. Substituting the transformations given in equation (9), the above equation is obtained as:

$$
N u_{x} \frac{1}{\operatorname{Re}_{x}^{1 / 2}}=-\left(N r+\frac{\kappa_{n f}}{\kappa_{f}}\right) \theta^{\prime}(0)
$$

\section{Solution Approach}

Intending to numerically solve the system of non-linear ordinary differential equations (10)-(11) with assisting boundary conditions equation (12), Galerkin finite element method (FEM) Ali et al. (2019) is applied with utilization of Gauss elimination scheme. In order to deal with boundary conditions at infinity or exterior of momentum and energy boundary layers, $\eta_{\max }=6$ is assumed for computational purpose. The entire flow region is divided into 1000 linear elements of identical size $\Delta \eta=0.001$, where each element contains two nodes. Firstly, order of the equation (10) is reduced by employing $f^{\prime}=h$, therefore, the resulting coupled system of differential equations is obtained as follows:

$f^{\prime}=h$

$$
\frac{1}{(1-\phi)^{5 / 2}} h^{\prime \prime}+\left(1-\phi+\phi \frac{\rho_{C N T}}{\rho_{f}}\right)\left(f h^{\prime}-h^{2}\right)=0
$$




$$
\left(N r+\frac{\kappa_{n f}}{\kappa_{f}}\right) \theta^{\prime \prime}+\left[1-\phi+\phi \frac{\left(\rho C_{p}\right)_{C N T}}{\left(\rho C_{p}\right)_{f}}\right] \operatorname{Pr}\left(f \theta^{\prime}-h \theta\right)=0
$$

and the corresponding boundary conditions are:

$\eta=0: f=S, h=1+\delta h^{\prime}, \theta=1$

$\eta \rightarrow \infty: h \rightarrow 0, \theta \rightarrow 0$

The variational form associated with the equations (17)-(19) over an individual two-nodded element $\left(\eta_{c}, \eta_{c+1}\right)$ is stated as follows:

$$
\begin{aligned}
& \int_{\eta_{c}}^{\eta_{c+1}} \omega_{1}\left(f^{\prime}-h\right) d \eta=0 \\
& \int_{\eta_{c}}^{\eta_{c+1}} \omega_{2}\left[\frac{1}{(1-\phi)^{5 / 2}} h^{\prime \prime}+\left(1-\phi+\phi \frac{\rho_{C N T}}{\rho_{f}}\right)\left(f h^{\prime}-h^{2}\right)\right] d \eta=0 \\
& \int_{\eta_{c}}^{\eta_{c+1}} \omega_{3}\left\{\left(N r+\frac{\kappa_{n f}}{\kappa_{f}}\right) \theta^{\prime \prime}+\left[1-\phi+\phi \frac{\left(\rho C_{p}\right)_{C N T}}{\left(\rho C_{p}\right)_{f}}\right] \operatorname{Pr}\left(f \theta^{\prime}-h \theta\right)\right\} d \eta=0
\end{aligned}
$$

here $\omega_{1}, \omega_{2}$ and $\omega_{3}$ are the weight functions corresponding to the functions $f, h$ and $\theta$. Substituting the following finite element approximations in equations (21)-(23), to obtain the finite element model

$$
f=\sum_{j=1}^{2} f_{j} \psi_{j}, \quad h=\sum_{j=1}^{2} h_{j} \psi_{j}, \quad \theta=\sum_{j=1}^{2} \theta_{j} \psi_{j} .
$$

with $\omega_{1}=\omega_{2}=\omega_{3}=\psi_{i},(i=1,2)$, where $\psi_{i}$ are the shape functions for a typical $\mathrm{c}^{\text {th }}$ linear element $\left(\eta_{c}, \eta_{c+1}\right)$ and are taken as:

$$
\psi_{1}^{(c)}=\frac{\eta_{c+1}-\eta}{\eta_{c+1}-\eta_{c}}, \quad \psi_{2}^{(c)}=\frac{\eta-\eta_{c}}{\eta_{c+1}-\eta_{c}}, \quad \eta_{c} \leq \eta \leq \eta_{c+1}
$$

Therefore, the finite element model of the equations (21)-(23) for $\mathrm{c}^{\text {th }}$ element is formulated as: 


$$
\left[\begin{array}{lll}
{\left[H^{11}\right]} & {\left[H^{12}\right]} & {\left[H^{13}\right]} \\
{\left[H^{21}\right]} & {\left[H^{22}\right]} & {\left[H^{23}\right]} \\
{\left[H^{31}\right]} & {\left[H^{32}\right]} & {\left[H^{33}\right]}
\end{array}\right]\left[\begin{array}{l}
\{f\} \\
\{h\} \\
\{g\}
\end{array}\right]=\left[\begin{array}{l}
\left\{b^{1}\right\} \\
\left\{b^{2}\right\} \\
\left\{b^{3}\right\}
\end{array}\right] .
$$

where $\left[H^{m n}\right]$ and $\left[b^{m}\right](m=1,2,3 \& n=1,2,3)$ are given by

$$
\begin{gathered}
H_{i j}^{11}=\int_{\eta_{c}}^{\eta_{c+1}} \psi_{i} \frac{d \psi_{i}}{d \eta} d \eta, H_{i j}^{12}=-\int_{\eta_{c}}^{\eta_{c+1}} \psi_{i} \psi_{j} d \eta, H_{i j}^{13}=0, \\
H_{i j}^{21}=0, H_{i j}^{22}=\int_{\eta_{c}}^{\eta_{c+1}}\left[-\frac{1}{(1-\phi)^{5 / 2}} \frac{d \psi_{i}}{d \eta} \frac{d \psi_{j}}{d \eta}+\left(1-\phi+\phi \frac{\rho_{s}}{\rho_{f}}\right)\left(\bar{f} \psi_{i} \frac{d \psi_{i}}{d \eta}-\bar{h} \psi_{i} \psi_{j}\right)\right] d \eta, H_{i j}^{23}=0, \\
H_{i j}^{31}=0, H_{i j}^{32}=0, \\
H_{i j}^{33}=\int_{\eta_{c}}^{\eta_{c+1}}\left\{-\left(N r+\frac{\kappa_{n f}}{\kappa_{f}}\right) \frac{d \psi_{i}}{d \eta} \frac{d \psi_{j}}{d \eta}+\left[1-\phi+\phi \frac{\left(\rho C_{p}\right)_{s}}{\left(\rho C_{p}\right)_{f}}\right] \operatorname{Pr}\left(\bar{f} \psi_{i} \frac{d \psi_{i}}{d \eta}-\bar{h} \psi_{i} \psi_{j}\right)\right\} d \eta, \\
b_{i}^{1}=0, b_{i}^{2}=-\frac{1}{(1-\phi)^{5 / 2}}\left(\psi_{i} \frac{d h}{d \eta}\right)_{\eta_{c}}^{\eta_{c+1}} \text { and } b_{i}^{3}=-\left(N r+\frac{\kappa_{n f}}{\kappa_{f}}\right)\left(\psi_{i} \frac{d \theta}{d \eta}\right)_{\eta_{c}}^{\eta_{c+1}} .
\end{gathered}
$$

where, $\bar{f}=\sum_{i=1}^{2} \bar{f}_{i} \psi_{i}$, and $\bar{h}=\sum_{i=1}^{2} \bar{h}_{i} \psi_{i}$ are supposed to be known.

The matrix constructed by assemblage of element equations, is of order $3003 \times 3003$. It holds a system of linear equation, which is to be solved by employing a direct or indirect iterative process. Subsequently, implementing the boundary conditions, the system of remaining 2998 equations can be evaluated by Newton-Raphson technique. The process is terminated when the solution is converged with desired accuracy $10^{-7}$, i.e.,

$$
\sum_{i}\left|\Omega_{i}^{j+1}-\Omega_{i}^{j}\right| \leq 10^{-7}
$$

where, $\Omega$ refers either $f, h$ or $\theta$ and $j$ is representing the iterative step.

\section{Validation of Results}

Table 2 represents the comparison made with the values by Mahdy (2012) and Jafari and Freidoonimehr (2015) to validate the present results of heat transfer rate $\theta^{\prime}(0)$ for different values of Prandtl number. 
International Journal of Mathematical, Engineering and Management Sciences

Vol. 6, No. 3, 860-877, 2021

https://doi.org/10.33889/IJMEMS.2021.6.3.051

Table 2. Comparison of present results for $-\theta^{\prime}(0)$ with previously published data when $S=0=\delta=\phi$ and

$$
f^{\prime \prime}(0)=-1.00048312 \text {. }
$$

\begin{tabular}{|c|c|c|c|}
\hline Pr & Mahdy(2012) & $\begin{array}{c}\text { Jafari and Freidoonimehr } \\
(2015)\end{array}$ & $\begin{array}{c}\text { Present results } \\
(\eta \rightarrow \infty=6)\end{array}$ \\
\hline 0.72 & 0.80868 & 0.80863135 & 0.812066360 \\
\hline 1.00 & 1.00000 & 1.00000000 & 1.000483120 \\
\hline 3.00 & 1.92368 & 1.92368259 & 1.923454906 \\
\hline 7.00 & 3.07224 & 3.07225021 & 3.072079370 \\
\hline
\end{tabular}

\section{Results and Discussion}

In this portion, an attempt is made to analyze the impact of controlling parameters, namely, solid volume fraction $(\phi)$, mass flux parameter $(S)$ and velocity slip parameter $(\delta)$ on both velocity and temperature profiles via Figures 2-8. Further, variations in skin friction parameter and Nusselt number with diverse values of aforesaid parameters have been examined through data displayed in tabulated form. It is notable that all other parameters are considered as constant while observing one parameter.

Impact of uniform mass flux parameters on dimensionless velocity profile is illustrated in Figure 2. The flow velocity is observed to be decreased due to increasing effect of suction while the reversal response is recorded for increasing injection effect. This is an outcome of the fact that boundary layer gets more close to the surface which in result destroys the momentum, which leads to fall in fluid velocity. Figure 3 shows the responses of dimensionless temperature profiles to mass flux parameter. It is analyzed that temperature as well as thermal boundary layer thickness are decreasing for larger values of suction/injection parameter.

The investigation of velocity slip parameter on velocity and temperature profiles in presence of suction effect is demonstrated in Figures 4 and 5 respectively. A reduction for $\eta<2.8$ and a slight growth for $\eta>2.8$ is detected in the fluid flow for boosting values of slip parameter. The slip boundary condition is implemented when the impact of fluid viscosity at the surface is negligible. Since slip effect causes weaker bond between the surface and fluid as more resistance is experienced in conveyance of velocity of stretching sheet to the fluid flow, therefore, it decreases the velocity. Further, Figure 5 reveals that higher values of velocity slip parameter correspond to rise in temperature throughout the boundary layer.

Figures 6 and 7 exhibit the effect of solid volume fraction parameter on dimensionless momentum and thermal profiles respectively. It is found that velocity distribution is an increasing function of solid volume fraction parameter for SWCNTs. From a physical perspective, enhancement in nanoparticle volume fraction gives rise to convective flow. Further, nanomaterial volume fraction has a significant impact on temperature field. From Figure 7 it is observed that temperature profile also shows the increasing behavior for higher solid volume fraction. It is apparent that thermal conductivity and diffusivity increases when volume of nanoparticles is increased. Hence, improvement in heat transfer leads to rise in temperature.

Figure 8 portrays the outcome of thermal radiation parameter on temperature profiles and it shows that higher values of radiation parameter enhance the fluid temperature. This is evident due to the fact that increment in radiation parameter decreases the Rosseland radiative mean 
International Journal of Mathematical, Engineering and Management Sciences

Vol. 6, No. 3, 860-877, 2021

https://doi.org/10.33889/IJMEMS.2021.6.3.051

absorptivity $k^{*}$ which leads to upsurge in radiative heat flux as $q_{r}=-\frac{4 \sigma^{*}}{3 k^{*}} \frac{\partial T^{4}}{\partial y}$. Hence increasing rate of radiative heat flux enlarges thickness of thermal boundary layer as well as temperature.

Table 3 shows the effect of mass flux parameter $(S)$, velocity slip parameter $\delta$ and nanoparticle volume fraction parameter $(\phi)$ on the shear stress $f^{\prime \prime}(0)$ and heat transfer rate $\theta^{\prime}(0)$ of SWCNT-kerosene oil nanofluid at the surface by considering other parameters as constant. From equations (14) and (16), it is evident that $C_{f} \operatorname{Re}_{x}^{1 / 2}$ and $N u_{x} \frac{1}{\operatorname{Re}_{x}^{1 / 2}}$ are directly proportional to the surface shear stress $f^{\prime \prime}(0)$ and heat transfer rate $\theta^{\prime}(0)$ respectively. Therefore, it can be concluded that local skin friction and local Nusselt number are amplifying for larger values of $\delta$ and $\phi$, while contrasting occurrence is observed for higher values of $S$. Further, surface heat flux is observed to be increased with increasing values of $\mathrm{Nr}$.

Table 3. Computational values of $f^{\prime \prime}(0)$ and $\theta^{\prime}(0)$ with variation in different physical parameters at $\operatorname{Pr}=21$.

\begin{tabular}{|c|c|c|c|c|c|}
\hline$S$ & $\delta$ & $\phi$ & $N r$ & $-f^{\prime \prime}(0)$ & $-\theta^{\prime}(0)$ \\
\hline-0.30 & 0.1 & 0.10 & 1.0 & 0.7455156 & 1.7052217 \\
\hline-0.15 & & & & 0.7969034 & 2.0257015 \\
\hline 0.00 & & & & 0.8519872 & 2.3992670 \\
\hline 0.15 & & & & 0.9107100 & 2.8225749 \\
\hline 0.30 & & & & 0.9729390 & 3.2904760 \\
\hline \multirow[t]{12}{*}{0.15} & 0.6 & & & 0.5694258 & 2.4701928 \\
\hline & 1.1 & & & 0.4222573 & 2.2732250 \\
\hline & 1.6 & & & 0.3381182 & 2.1391947 \\
\hline & 2.1 & & & 0.2830635 & 2.0390050 \\
\hline & 0.1 & 0.05 & & 0.9262844 & 3.4890080 \\
\hline & & 0.15 & & 0.8884413 & 2.3684170 \\
\hline & & 0.20 & & 0.8601833 & 2.0334220 \\
\hline & & 0.25 & & 0.8265376 & 1.7731068 \\
\hline & & 0.10 & 3.0 & 0.9107100 & 2.2160010 \\
\hline & & & 5.0 & & 1.8607720 \\
\hline & & & 7.0 & & 1.6215969 \\
\hline & & & 9.0 & & 1.4469150 \\
\hline
\end{tabular}


International Journal of Mathematical, Engineering and Management Sciences

Vol. 6, No. 3, 860-877, 2021

https://doi.org/10.33889/IJMEMS.2021.6.3.051

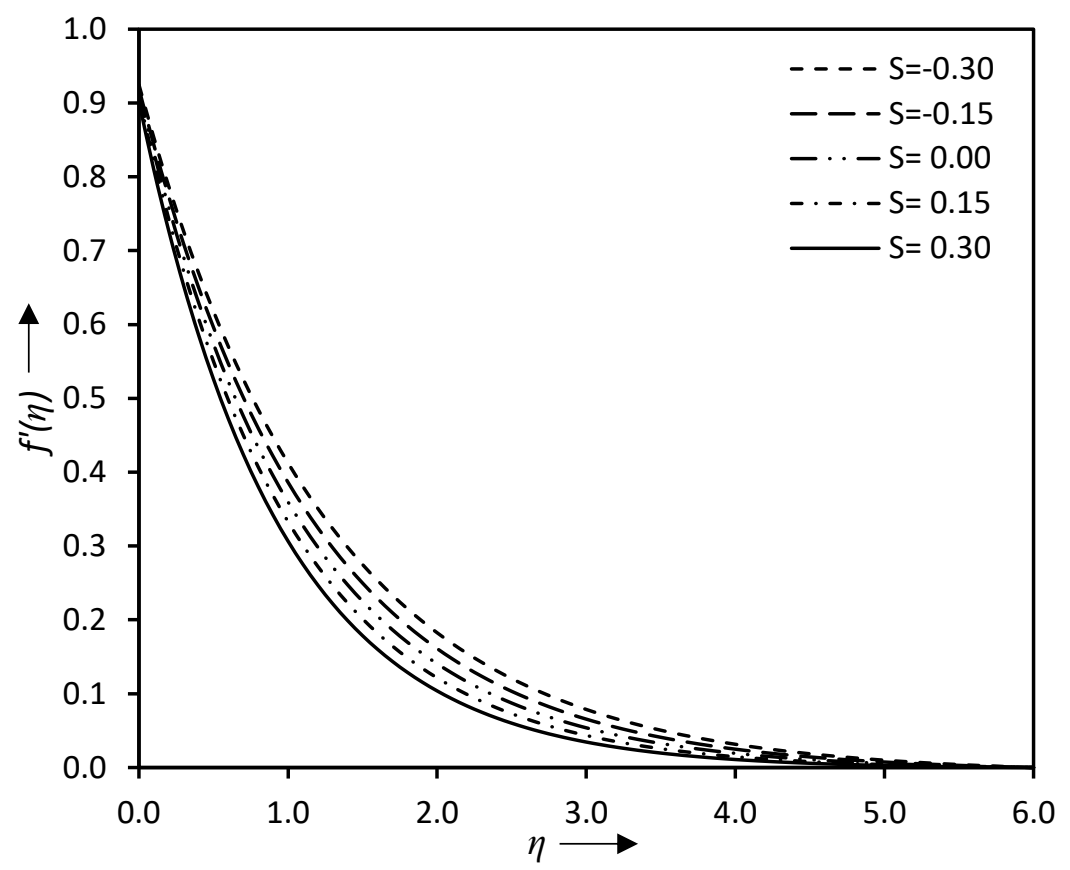

Figure 2. Velocity profiles for various values of $S$ at $\delta=0.1$ and $\phi=0.1$.

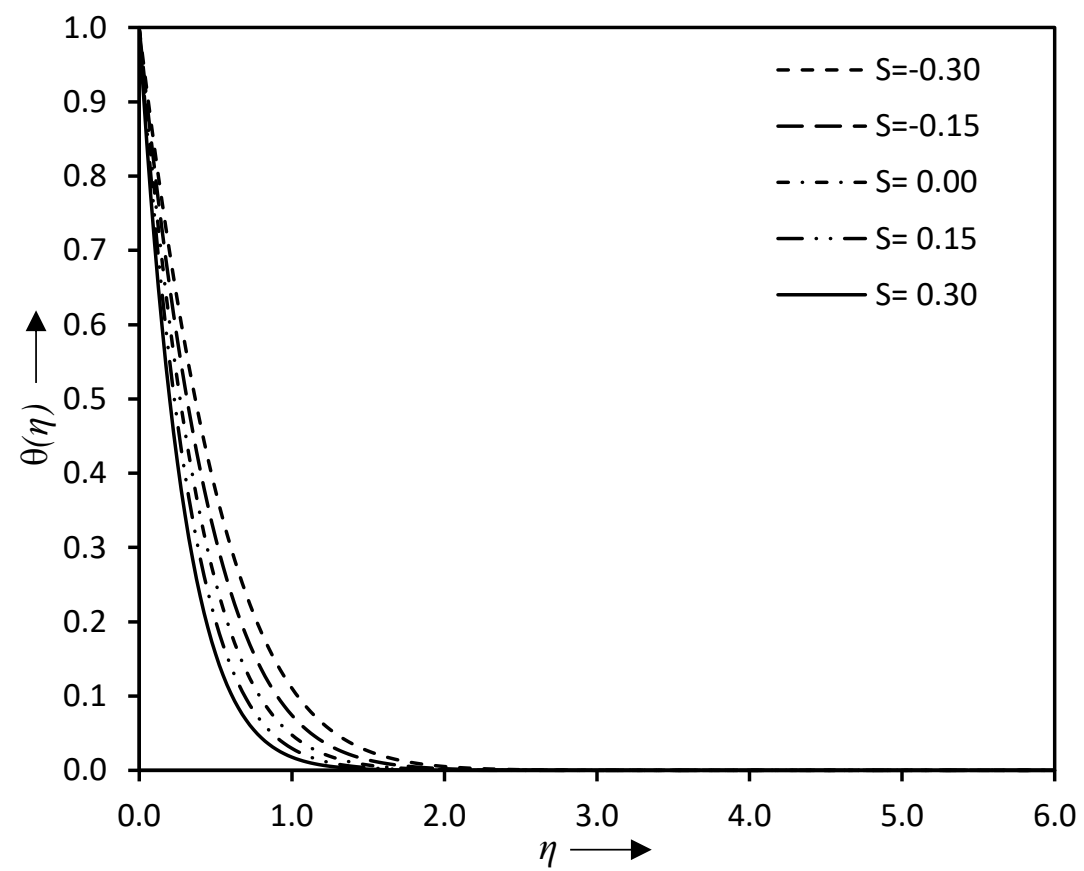

Figure 3. Temperature profiles for various values of $S$ at $\delta=0.1, \phi=0.1$ and $\operatorname{Pr}=21$. 
International Journal of Mathematical, Engineering and Management Sciences

Vol. 6, No. 3, 860-877, 2021

https://doi.org/10.33889/IJMEMS.2021.6.3.051



Figure 4. Velocity profiles for various values of $\delta$ at $S=0.15$ and $\phi=0.1$.

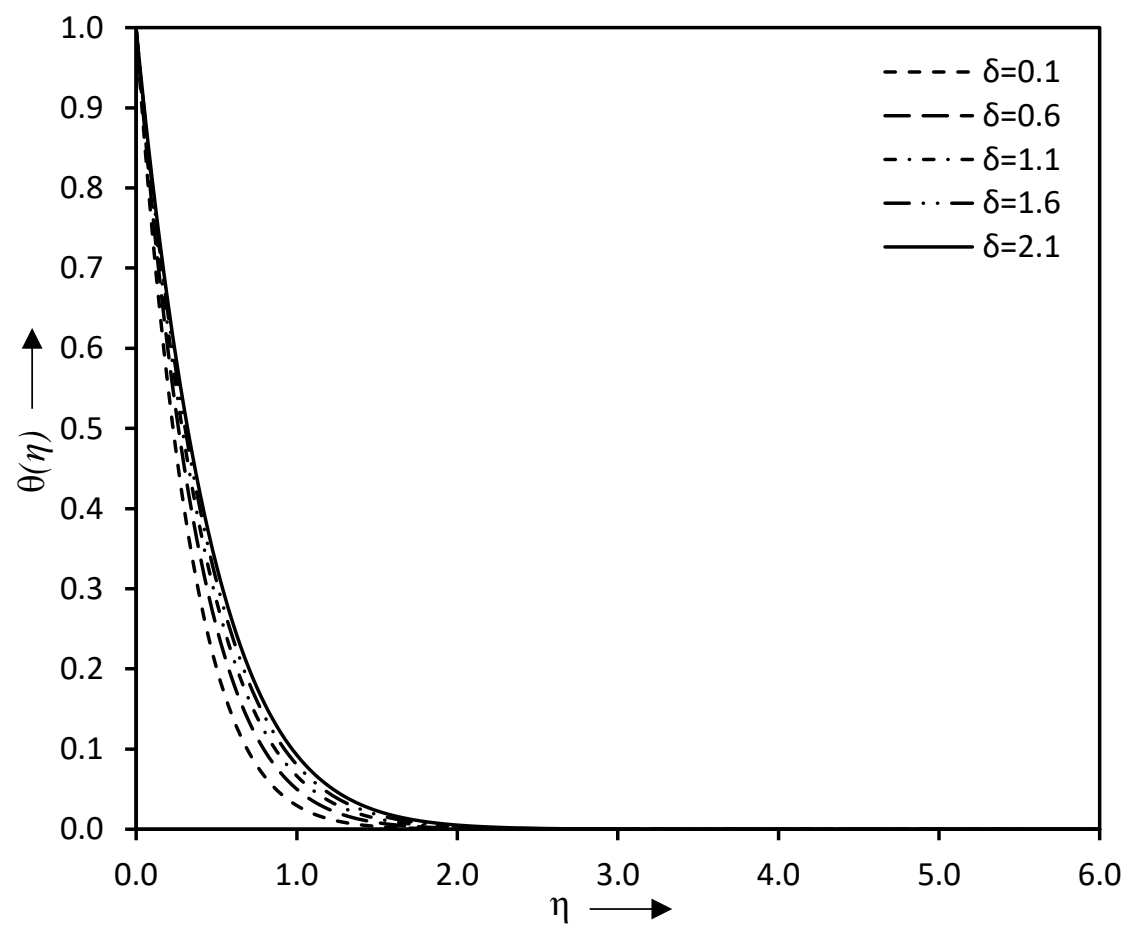

Figure 5. Temperature profiles for various values of $\delta$ at $S=0.15, \phi=0.1$ and $\operatorname{Pr}=21$. 
International Journal of Mathematical, Engineering and Management Sciences

Vol. 6, No. 3, 860-877, 2021

https://doi.org/10.33889/IJMEMS.2021.6.3.051

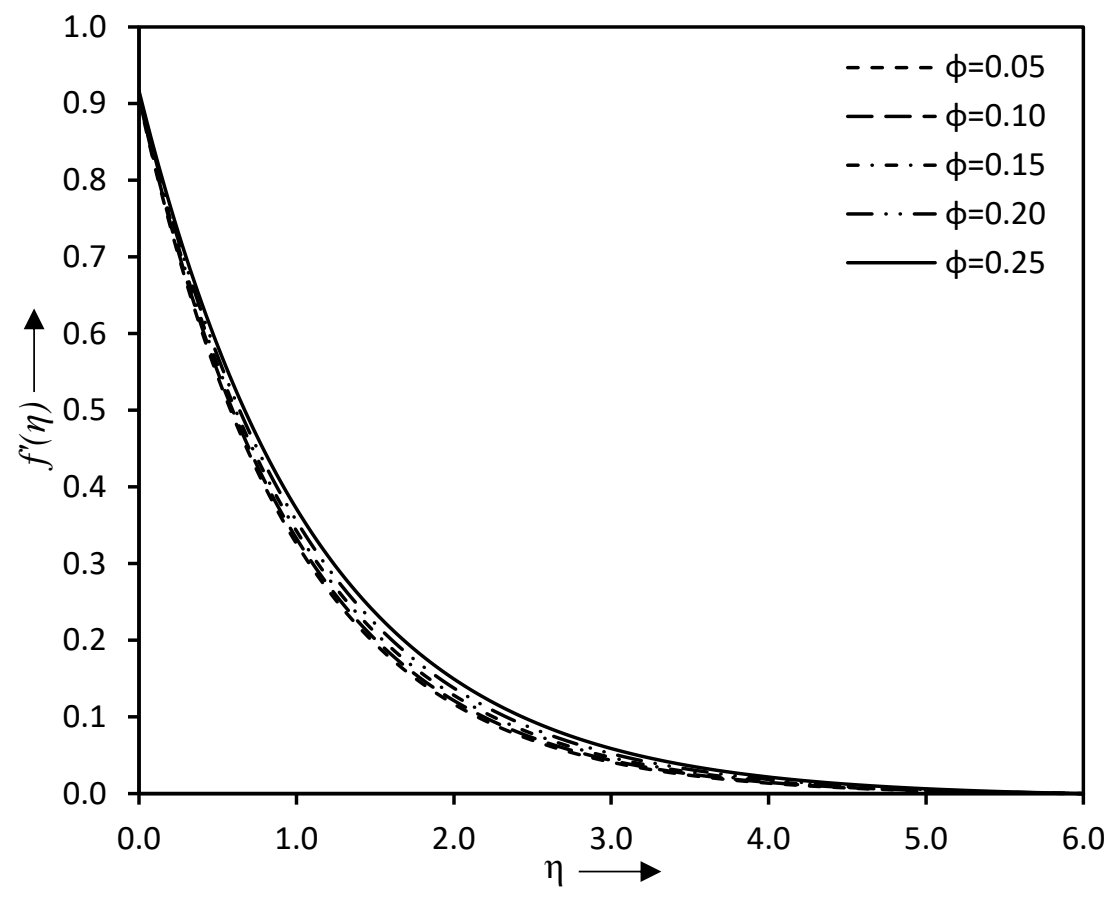

Figure 6. Velocity profiles for various values of $\phi$ at $S=0.15$ and $\delta=0.1$.

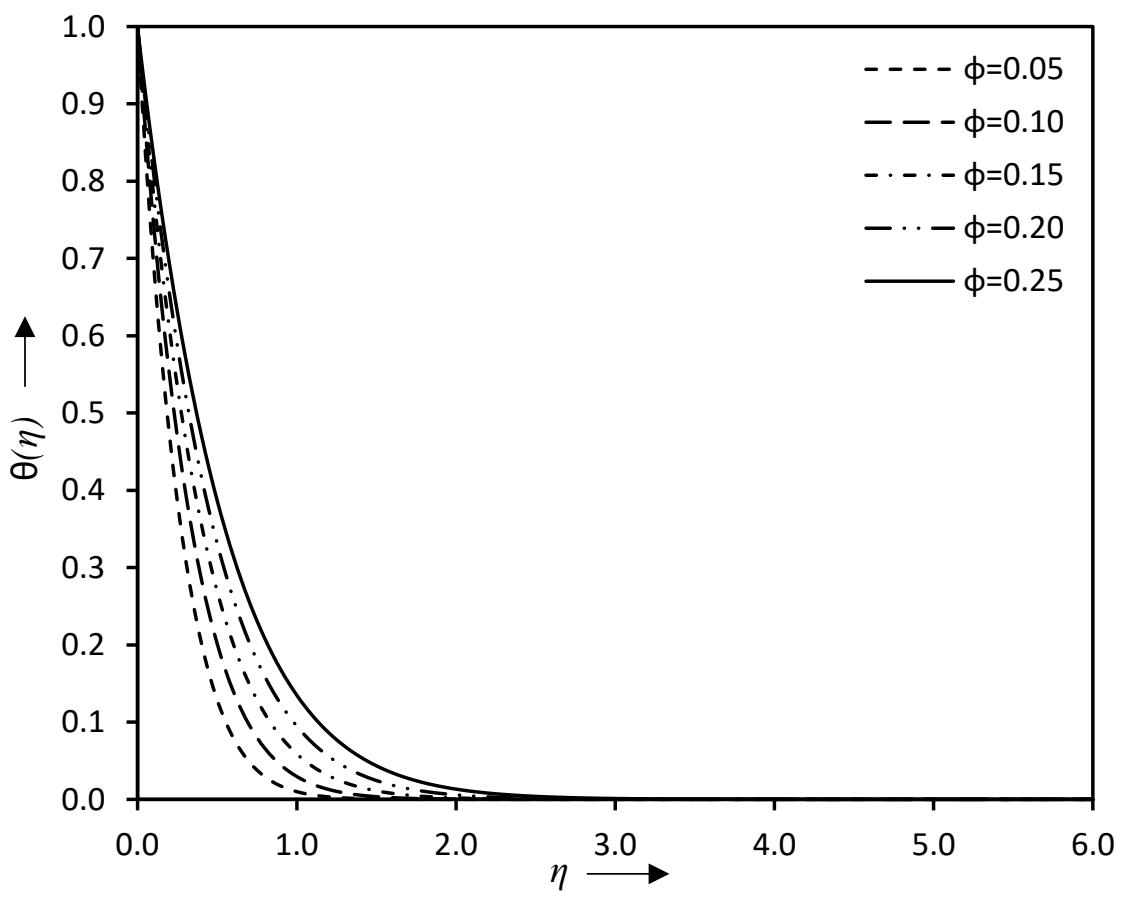

Figure 7. Temperature profiles for various values of $\phi$ at $S=0.15, \delta=0.1$ and $\operatorname{Pr}=21$. 


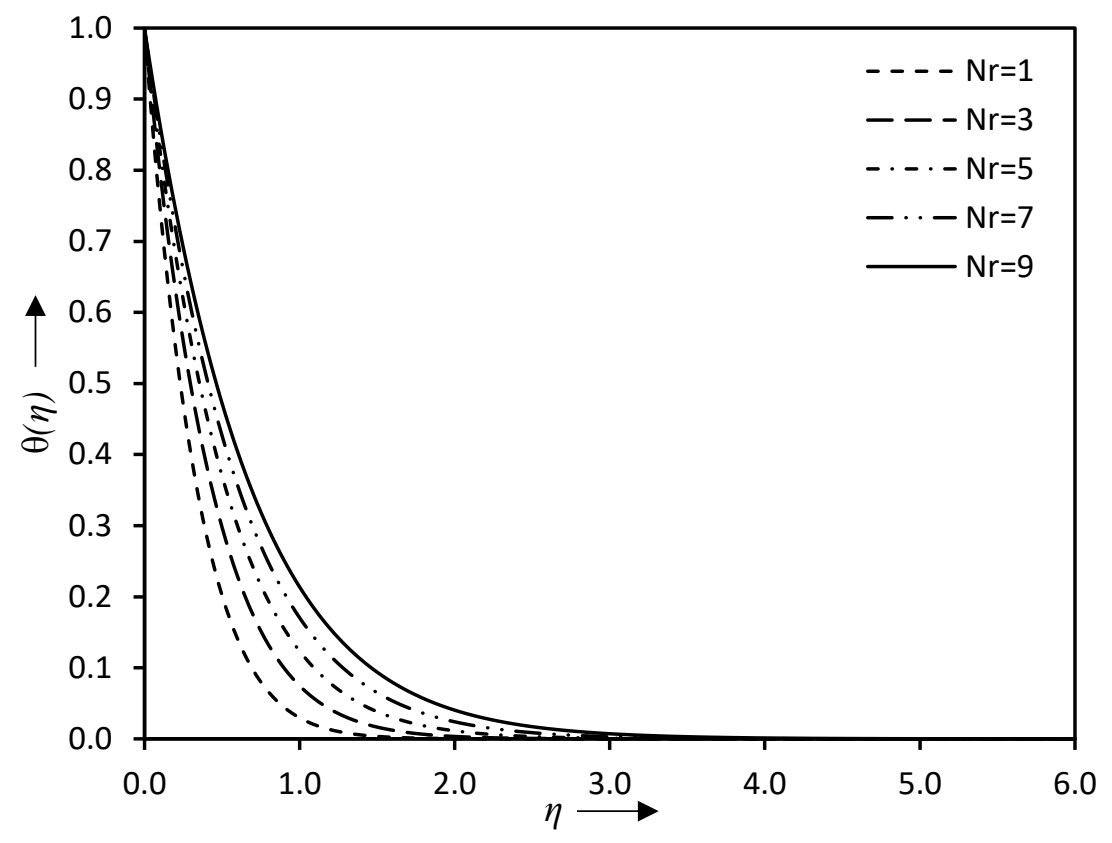

Figure 8. Temperature profiles for various values of $N r$ at $S=0.15, \delta=0.1$ and $\operatorname{Pr}=21$.

\section{Conclusions}

Flow of SWCNT-kerosene oil nanofluid over a linear stretching surface has been addressed in appearance of velocity slip and suction/injection effects. Finite element method is employed to solve the governing non-linear ordinary differential equations. The main outcomes of this problem can be summarized as follows:

- $\quad$ Fluid velocity, surface shear stress, temperature and surface heat flux are all increasing functions of solid volume fraction parameter and decreasing functions of mass flux parameter.

- $\quad$ Thickness of momentum boundary layer reduces in the range $\eta<2.8$, whereas thermal boundary layer thickness, surface heat flux as well surface shear stress increases for large values of velocity slip parameter.

- Local Nusselt number and temperature both enhances with increment in radiation parameter.

\section{Conflict of Interest}

Authors declare that they have no conflict of interest.

\section{Acknowledgements}

Authors would like to acknowledge University Grant commission (UGC), New Delhi for financial support under the Grant No. F. No. 16-6(DEC.2018)/2019(NET/CSIR) and UGC-Ref. No. 1202 on 02-04-2019. 
International Journal of Mathematical, Engineering and Management Sciences

Vol. 6, No. 3, 860-877, 2021

https://doi.org/10.33889/IJMEMS.2021.6.3.051

\section{References}

Afify, A.A. (2017). The influence of slip boundary condition on Casson nanofluid flow over a stretching sheet in the presence of viscous dissipation and chemical reaction. Mathematical Problems in Engineering, 2017, 1-12.

Akbar, N.S., Khan, Z.H., \& Nadeem, S. (2014). The combined effects of slip and convective boundary conditions on stagnation-point flow of CNT suspended nanofluid over a stretching sheet. Journal of Molecular Liquids, 196, 21-25.

Akbar, N.S., Nadeem, S., Haq, R.U., \& Khan, Z.H. (2013). Radiation effects on MHD stagnation point flow of nano fluid towards a stretching surface with convective boundary condition. Chinese Journal of Aeronautics, 26(6), 1389-1397.

Ali, L., Liu, X., Ali, B., Mujeed, S., \& Abdal, S. (2019). Finite element simulation of multi-slip effects on unsteady MHD bioconvective micropolar nanofluid flow over a sheet with solutal and thermal convective boundary conditions. Coatings, 9(12), 842 .

Attia, H.A. (2007). On the effectiveness of ion slip and uniform suction or injection on steady MHD flow due to a rotating disk with heat transfer and Ohmic heating. Chemical Engineering Communications, 194(10), 1396-1407.

Azzam, G.E.D.A. (2002). Radiation effects on the MHD mixed free-forced convective flow past a semiinfinite moving vertical plate for high temperature differences. Physica Scripta, 66(1), 71.

Chaudhary, S., \& Kanika, K.M. (2019). Radiation heat transfer on SWCNT and MWCNT based magnetohydrodynamic nanofluid flow with marangoni convection. Physica Scripta, 95(2), 025202.

Chaudhary, S., \& Kanika, K.M. (2020). Heat generation/absorption and radiation effects on hydromagnetic stagnation point flow of nanofluids toward a heated porous stretching/shrinking sheet with suction/injection. Journal of Porous Media, 23(1), 27-49.

Choi, S.U.S, \& Eastman, J.A. (1995). Enhancing thermal conductivity of fluids with nanoparticles. Technical report, Argonne National Lab., IL (United States).

Cortell, R. (2005). Flow and heat transfer of a fluid through a porous medium over a stretching surface with internal heat generation/absorption and suction/blowing. Fluid Dynamics Research, 37(4), 231.

Crane, L.J. (1970). Flow past a stretching plate. Zeitschrift f'ur angewandte Mathematik und Physik ZAMP, $21(4), 645-647$.

Dogonchi, A.S., \& Ganji, D.D. (2017). Impact of cattaneo-christov heat flux on MHD nanofluid flow and heat transfer between parallel plates considering thermal radiation effect. Journal of the Taiwan Institute of Chemical Engineers, 80, 52-63.

Eid, M.R., \& Makinde, O.D. (2018). Solar radiation effect on a magneto nanofluid flow in a porous medium with chemically reactive species. International Journal of Chemical Reactor Engineering, 16(9).

El-Arabawy, H.A.M. (2003). Effect of suction/injection on the flow of a micropolar fluid past a continuously moving plate in the presence of radiation. International Journal of Heat and Mass Transfer, 46(8), 1471-1477.

Ghalandari, M., Maleki, A., Haghighi, A., Shadloo, M.S., Nazari, M.A., \& Tlili, I. (2020). Applications of nanofluids containing carbon nanotubes in solar energy systems: a review. Journal of Molecular Liquids, 313, 113476.

Ghosh, S., \& Mukhopadhyay, S. (2019). Effects of slip on $\mathrm{Cu}$-water or $\mathrm{Fe}_{3} \mathrm{O}_{4}$-water nanofluid flow over an exponentially stretched sheet. Pramana, 92(6), 93. 
International Journal of Mathematical, Engineering and Management Sciences

Vol. 6, No. 3, 860-877, 2021

https://doi.org/10.33889/IJMEMS.2021.6.3.051

Gireesha, B.J., Mahanthesh, B., Gorla, R.S.R., \& Manjunatha, P.T. (2016). Thermal radiation and Hall effects on boundary layer flow past a non-isothermal stretching surface embedded in porous medium with non-uniform heat source/sink and fluid particle suspension. Heat and Mass Transfer, 52(4), 897911.

Hady, F.M., Ibrahim, F.S., Abdel-Gaied, S.M., \& Eid, M.R. (2012). Radiation effect on viscous flow of a nanofluid and heat transfer over a nonlinearly stretching sheet. Nanoscale Research Letters, 7(1), 229.

Haq, R.U., Nadeem, S., Khan, Z.H., \& Noor, N.F.M. (2015). Convective heat transfer in MHD slip flow over a stretching surface in the presence of carbon nanotubes. Physica B: Condensed Matter, 457, 4047.

Hayat, T., Hussain, Z., Alsaedi, A., \& Asghar, S. (2016). Carbon nanotubes effects in the stagnation point flow towards a nonlinear stretching sheet with variable thickness. Advanced Powder Technology, 27(4), 1677-1688.

Hayat, T., Qasim, M., \& Mesloub, S. (2011). MHD flow and heat transfer over permeable stretching sheet with slip conditions. International Journal for Numerical Methods in Fluids, 66(8), 963-975.

Jafari, S.S., \& Freidoonimehr, N. (2015). Second law of thermodynamics analysis of hydro-magnetic nanofluid slip flow over a stretching permeable surface. Journal of the Brazilian Society of Mechanical Sciences and Engineering, 37(4), 1245-1256.

Jang, J., \& Wereley, S.T. (2004). Pressure distributions of gaseous slip flow in straight and uniform rectangular microchannels. Microfluidics and Nanofluidics, 1(1), 41-51.

Kandasamy, R., Loganathan, P., \& Arasu, P.P. (2011). Scaling group transformation for MHD boundarylayer flow of a nanofluid past a vertical stretching surface in the presence of suction/injection. Nuclear Engineering and Design, 241(6), 2053-2059.

Kanika, K., Chaudhary, S., \& Choudhary, M.K. (2020). Influence of magnetic field on thermal radiation and particle shapes of copper-water nanofluid considering marangoni boundary layer. International Journal of Mathematical, Engineering and Management Sciences, 5(5), 957-970.

Khan, Z.H., Hussain, S.T., Hammouch, Z., \& Haq, R.U. (2016). Flow and heat transfer analysis of water and ethylene glycol based $\mathrm{Cu}$ nanoparticles between two parallel disks with suction/injection effects. Journal of Molecular Liquids, 221, 298-304.

Krishna, P.M., Ramreddy, C., \& Rao, C.V. (2019). Effects of double stratification on MHD flow and heat transfer of nanofluid along a permeable vertical plate. International Journal of Mathematical, Engineering and Management Sciences, 4(6), 1362-1372.

Kumaresan, V., \& Velraj, R. (2012). Experimental investigation of the thermo-physical properties of water-ethylene glycol mixture based CNT nanofluids. Thermochimica Acta, 545, 180-186.

Liu, Z.H., \& Liao, L. (2010). Forced convective flow and heat transfer characteristics of aqueous dragreducing fluid with carbon nanotubes added. International Journal of Thermal Sciences, 49(12), 23312338.

Mahdy, A. (2012). Unsteady mixed convection boundary layer flow and heat transfer of nanofluids due to stretching sheet. Nuclear Engineering and Design, 249, 248-255.

Makinde, O.D., \& Chinyoka, T. (2013). Numerical investigation of buoyancy effects on hydromagnetic unsteady flow through a porous channel with suction/injection. Journal of Mechanical Science and Technology, 27(5), 1557-1568.

Mishra, A., \& Kumar, M. (2020). Velocity and thermal slip effects on MHD nanofluid flow past a stretching cylinder with viscous dissipation and joule heating. SN Applied Sciences, 2(8), 1-13. 
International Journal of Mathematical, Engineering and Management Sciences

Vol. 6, No. 3, 860-877, 2021

https://doi.org/10.33889/IJMEMS.2021.6.3.051

Mohammadein, S.A., Raslan, K., Abdel-Wahed, M.S., \& Abedel-Aal, E.M. (2018). KKL model of MHD $\mathrm{CuO}$-nanofluid flow over a stagnation point stretching sheet with nonlinear thermal radiation and suction/injection. Results in Physics, 10, 194-199.

Mukhopadhyay, S., \& Andersson, H.I. (2009). Effects of slip and heat transfer analysis of flow over an unsteady stretching surface. Heat and Mass Transfer, 45(11), 1447-1452.

Mukhopadhyay, S., \& Layek, G.C. (2008). Effects of thermal radiation and variable fluid viscosity on free convective flow and heat transfer past a porous stretching surface. International Journal of Heat and Mass Transfer, 51(9-10), 2167-2178.

Pantokratoras, A., \& Fang, T. (2012). Sakiadis flow with nonlinear rosseland thermal radiation. Physica Scripta, 87(1), 015703.

Rad, P.M., \& Aghanajafi, C. (2009). The effect of thermal radiation on nanofluid cooled microchannels. Journal of Fusion Energy, 28(1), 91-100.

Shaw, S., Kameswaran, P.K., \& Sibanda, P. (2016). Effects of slip on nonlinear convection in nanofluid flow on stretching surfaces. Boundary Value Problems, 2016(1), 1-11.

Sheikholeslami, M., \& Ganji, D.D. (2015). Unsteady nanofluid flow and heat transfer in presence of magnetic field considering thermal radiation. Journal of the Brazilian Society of Mechanical Sciences and Engineering, 37(3), 895-902.

Tulu, A., \& Ibrahim, W. (2020). MHD slip flow of CNT-ethylene glycol nanofluid due to a stretchable rotating disk with Cattaneo-Christov heat flux model. Mathematical Problems in Engineering, 2020, $1-13$.

Xie, H., Lee, H., Youn, W., \& Choi, M. (2003). Nanofluids containing multiwalled carbon nanotubes and their enhanced thermal conductivities. Journal of Applied Physics, 94(8), 4967-4971. 